\title{
EFFECTIVE COVERAGE AND CONNECTIVITY CONSERVATION WITH LOAD BALANCING FOR WSN
}

\author{
MohammedJameel A Zarzari ${ }^{1}$, Bindhu Madhavi $\mathbf{P}^{2}$, M. Nagendra ${ }^{3}$ \\ ${ }^{1}$ Student, Department of CSE, T. JIT, Karnataka, India \\ ${ }^{2}$ Associate Professor, Department of CSE, T. JIT, Karnataka, India \\ ${ }^{3}$ Associate Professor, Department of CSE, S. K. University, Karnataka, India
}

\begin{abstract}
Wireless sensor networks provide maximum coverage area in a network as much as possible, as the basic objective. Such as, identifying the location of interruptions in combat zone and tracking of an object, with reliable services. Organization of sensor nodes to construct maximum subgroups with energy limit, ability to monitor every required discrete points and activation in alternate manner is the best possible method for quality assurance. Behind the objective accomplishing the maximum subgroups, in the aim of obtaining the maximum connectivity arises the question on assurance of connectivity among nodes. To assure full coverage with base station connectivity in each sensing node, this paper proposes a new algorithm as Maximum Connected Load Balancing Cover Tree (MCLCT) with dynamically formation of routing cover tress with load-balancing. This kind of problem can be known as maximum cover tree problem, proved as nondeterministic polynomial-complete. There are two techniques in proposed MCLCT, first one is conservation of coverage by using recursive heuristic coverage optimization, and second is strategy for identification of route using probabilistic load balance. The proposed MCLCT provides the solution by sharing the load among the nodes while sensing and transmitting, hence this makes energy consumption balanced between the nodes. Collection of simulation results depicts that, the proposed method accomplishes the objectives in terms of conservation of connectivity and energy efficiency.
\end{abstract}

Keywords: WSN, nodes/hubs coverage/connectivity, and lifetime maximization.

\section{INTRODUCTION}

Wireless sensor nodes pursuing the functionality of sensing, manipulating and capacity of storing environmental information with capability of transmission of data to each node forms Wireless Sensor Networks (WSNs). These components permit WSN to be connected to an assortment of scope of utilizations, e.g. home medicinal services, front line reconnaissance, machine observing, ecological checking, etc. As of late, WSNs have likewise turned into a vital range of examination.

Basically, a wireless sensing node which has small batteries are located near the area which has to sense The activities taking place at the location which come under the sensing range provided by the sensor node. The every sensor node is equipped with a transceivers through which the link between the other nodes and base station is possible. In Wireless sensor network the data which is sensed by the node is transmitted to the sink node with energy efficient manner.
The main problem in WSN is the how well the DPOI are covered in sensing field and the coverage conservation is one of the big problem because the node has the minimum battery power and this we studies from different aspect. In papers [1],[2],[3] and [4], the nodes emplacement procedures is based on some role were used to locate optimal emplacement location. Before the nodes take its position in the environment coverage some task is to care out to achive the specific coverage. It is theoretical work made such that location of every sensing node is known after the sensing node is distributed in sensing field [5]-[7]. The scheduling is require to find the time of activating and deactivating the sensor node while managing the required coverage

\section{RELATED WORK}

In recently work, the finding of cover sets has moved to how to prepare the cover set which are connected to Base Station. It implies not just the complete placement of the sensor is spliced into a few cover set additionally steering ways for the dynamic sensor hubs in every cover set is contemplated. 
A broad arrangement of studies worried with the previously stated compound issue have been proposed [8]-[12]. For example, Cardie [9] define the Connected Set Covers issue that goes for finding a most extreme sets to which the nodes are directly connected and represent it as a cover set and the sensor node which is activated is to associated with the base station and represents its NP completeness. To adapt to the CSC issue, they utilized two incorporated calculation: an integer program base heuristic and greedy CSC. In studies [10], Ostovari shows the exhibited an appropriated strategy for connected point coverage. The hub could choose whether it can play a role as a data sensor node after a particular holding up time interim or go about as a relaying node in the modified-virtual-robust-spanning tree. Rather than utilizing the distance of the hubs to assess cost of hubs in the Minimum-spanning tree, MVRST took the jump include into record the assessment of edge expenses. Thought about with the methodology presented in [9], the creators guaranteed that the MVRST can cover all DPOIs utilizing small amount of sensor node and has a lower normal information misfortune rate.

Despite the fact that the previously stated studies [9]-[11] tended to the CSC issue, they utilized illogical and rearranged vitality utilization models which are a long way from the valid in WSNs. In [9]-[11], two vitality utilization examples were predefined for detecting and correspondence and did not include separations between hubs. That is, every one of the hubs serving as the detecting hubs/transferring hubs expend the same sum of vitality. Truth be told, energy utilization is depend on the size of information transmitting, the transmission distance between source and destination, the recurrence of occasion happened at DPOI, and the packet listening time. These components have to be consider when the energy utilization of WSN is assessed.

Receiving a suitable vitality utilization mode, gurusamy and Zhao [8] built up an estimate calculation with a dispersed variant, is given in Communication Weighted greedy cover, to maximize the quantity of set covers.. In particular, they built cover tree which consist of three properties: 1 . The each leaf node in tree is the sensor, 2) The root of tree is base station, and 3) Each DPOIs must have minimum one sensor node.

\section{PROBLEM DESCREPTION}

In this segment, the Maximum-Cover-Tree(MCT) issue is displayed as an optimization issue [8], [12]. In the meantime, we define the load of sensor hubs and present the considered accomplishing load balance among sensor hubs.

\subsection{The MCT Issue Formulation}

Let $\mathrm{P}$ represents the set of $\mathrm{m}$ DPOIs, where $\mathrm{P}=\{\mathrm{p} 1, \mathrm{p} 2, \ldots$, $\mathrm{pm}$ \} for $\mathrm{m}=|\mathrm{P}|$ and set $\mathrm{S}$ represents the $\mathrm{n}$ sensor nodes in WSNs, where $S=\{s 1, s 2, \ldots, s n\}$ for $n=|S| S=\{s 1, s 2, \ldots$ ., $\mathrm{Sn}\}$ for $\mathrm{n}=|\mathrm{S}|$. Such that every DPOIs in $\mathrm{P}$ is covered by minimum 1 sensor device from set $\mathrm{S}$ and energy EO is same for each node in network, Let consider the observation variables $\mathrm{Bi}, \mathrm{j}=1$ if DPOI $\mathrm{pj}$ is present in the range of sensing node si, generally $\mathrm{Bi}, \mathrm{j}$ is equal to zero. A graph $\mathrm{G}(\mathrm{V}, \mathrm{R})$ is un-directed which is delivered for the Wireless Sensor Network. The Base Station and all the sensor nodes are individuals from the arrangement of vertices $\mathrm{V}$ (i.e.., BS $\mathrm{U} \subseteq \mathrm{V}$ ) and two nodes ( $\mathrm{sj}$ and si) are said to be associated with an edge in $G$ in the event that their Euclidean separation di,j is less than or equal to the correspondence range $\mathrm{Rc}$ (i.e.., di,j $\leq \mathrm{Rc}$ ).

The developing element Cover-tree has 4 property as given below.

1. Sensor node which is always located in leaf of tree.

2. The root of the tree is represented as Base station.

3. Each DPOIs is covered by minimum 1 sensing node.

4. The sensor node cannot do both task i.e., relaying and sensing the data.

\subsection{Balancing the Load of Nodes in Network}

The balancing of load between the nodes is valuable if the network is distributed, for example the workload can be equally shared by sensor node in wireless sensor network. In wireless sensor networks, the load on sensor node can be measure by knowing the sensed data to be exchanged. The congestion in the network will take place if the node is heavily loaded because of which node drain its energy faster so the lifetime of network is reduced. So the load balancing is essential to increse the life time of network.

In cover tree created by MCLCT, every sensed information is to be transmitting through the relaying node to the base station straight forwardly or through a multi hub way, i.e., there is a transmission link between the sensing node and the base station by forming a tree like topology. For these dynamic-cover-trees, load adjusting can encourage the expansion of operational timeframe. Consequently, the proposed MCLCT frames the dynamic cover trees as well as finish the load equalization among sensor hubs. 


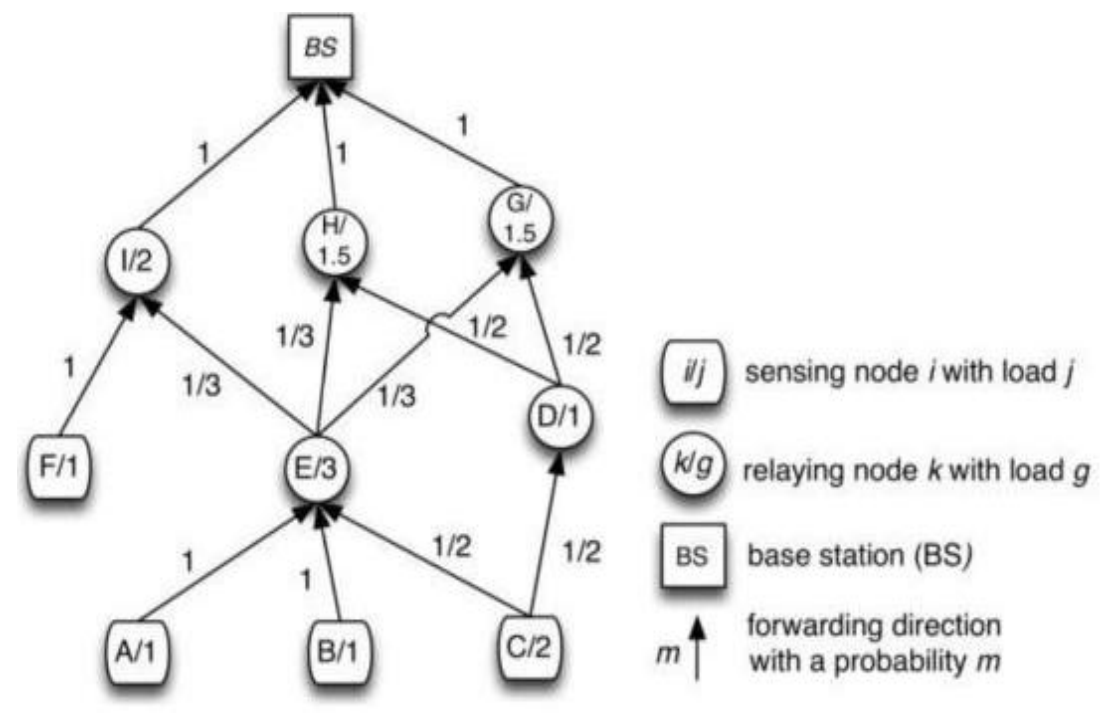

Fig -1 expected load calculation

\section{PROPOSED ALGORITHM}

\subsection{The Framework to resolve the MCT Issues.}

To build several number of Connected-cover-trees is the aim of MCT issue. By doing this the network lifetime will be maximized and can be possibly cover full range. The MCT issue is a complex NP-complete, so searching an imperfect arrangement is a nonspecific methodology keeping in mind the end goal to diminish the season of calculation. There are two sub parts in MCLCT 1) A Coverage-OptimizingRecursive (COR) and 2) Probable load balance.

Locating the extreme number of disjoint node which can accomplished by sink node is comes under COR-heuristic In every disjoint-sets, The nodes are capable of screen every DPOIs together. The coverage conservation issue is handled by the COR heuristic. To find the effective path from every single node to the Base Station is done by the PLB technique after the task of COR heuristic is completed. For every probable transmission path to candidate parent node, The different probabilities are assigned by the PLB strategy to provide uniform load, fig. 2 shows the flow diagram of given MCLCT algorithm.

To start with the group of disjoint sets (named as DS. P and $\mathrm{S}$ are introduced after a Wireless sensor network is given to the MCLCT(step1). At that point, the arrangement of accessible node, The nodes which are not covering any DPOI is come under the set $\mathrm{Sa}$ from $\mathrm{S}$ (step2).From $\mathrm{Sa}$, the OCR component will search the same number of disjoint set(Ci)of node as could be expected under the circumstances until the node in Sa can't be chosen to assembly another free disjoint set(step3 and step4).

The investigating process for disjoint set did by the MCLCT will isolate the arrangement of Sa into a few selective subset i.e., $\mathrm{Ci} \cap \mathrm{Cj}=\{\varnothing\}$, and $\mathrm{i}=\mathrm{j}$. Each disjoint set $\mathrm{Ci}$ of hubs can screen each single DPOIs at the same time. With a thorough of full coverage, after the investigation process, we can secure a gathering subsets of the accessible hubs ( $\mathrm{Sa}), \mathrm{DS}=$ $\{\mathrm{C} 1, \mathrm{C} 2, \ldots, \mathrm{Cw}\}$, which will be told to each part in DS.A short time later, we can get an arrangement of the leftover hubs by $\mathrm{Srsd}=\mathrm{S} \backslash \mathrm{DS}$.

The hubs in Srsd should be transformed the node into sleep mode to save the and a wake up message is send to nodes to change their state from sleep mode to active mode. In the following level, the arrangement of DS and Srsd are used to build relating dynamic cover tree.

Toward the start of this level, every component of $\{\tau 1, \tau 2$, . . . , $\tau \mathrm{w}\}$, is initialized to zero(step7). As earlier, each $\tau \mathrm{j}(\mathrm{w}=>\mathrm{j}=>1)$ is assessed per-time by figuring out if the developed tree like structure $\mathrm{T}(\tau \mathrm{j})$ can accomplish the overall coverage and base station connectivity(step9 to step18).Sensing node has to monitor every activity happening at the DPOI and must be notify to the base station.

The flow diagram of MCLCT to maintain overall coverage is shown in figure 4. A network which preforming the MCLCT operation will recover the failed coverage by bringing some sleeping node into active mode which is present in $\operatorname{Srsd}(\operatorname{step} 11)$

In the event of both full coverage (step12) also, BS connectivity of $\mathrm{T}(\mathrm{tj})$ (step13) are accomplished, the operational period $\mathrm{t} j$ will redesigned with an additional of one second(step14) Then again, if monitoring of fullcoverage is fails, that means either the full-coverage or BS connectivity is not met any longer,the as of now on obligation dynamic-cover-tree $\mathrm{T}(\mathrm{tj})$ will be inactivated(step16). 


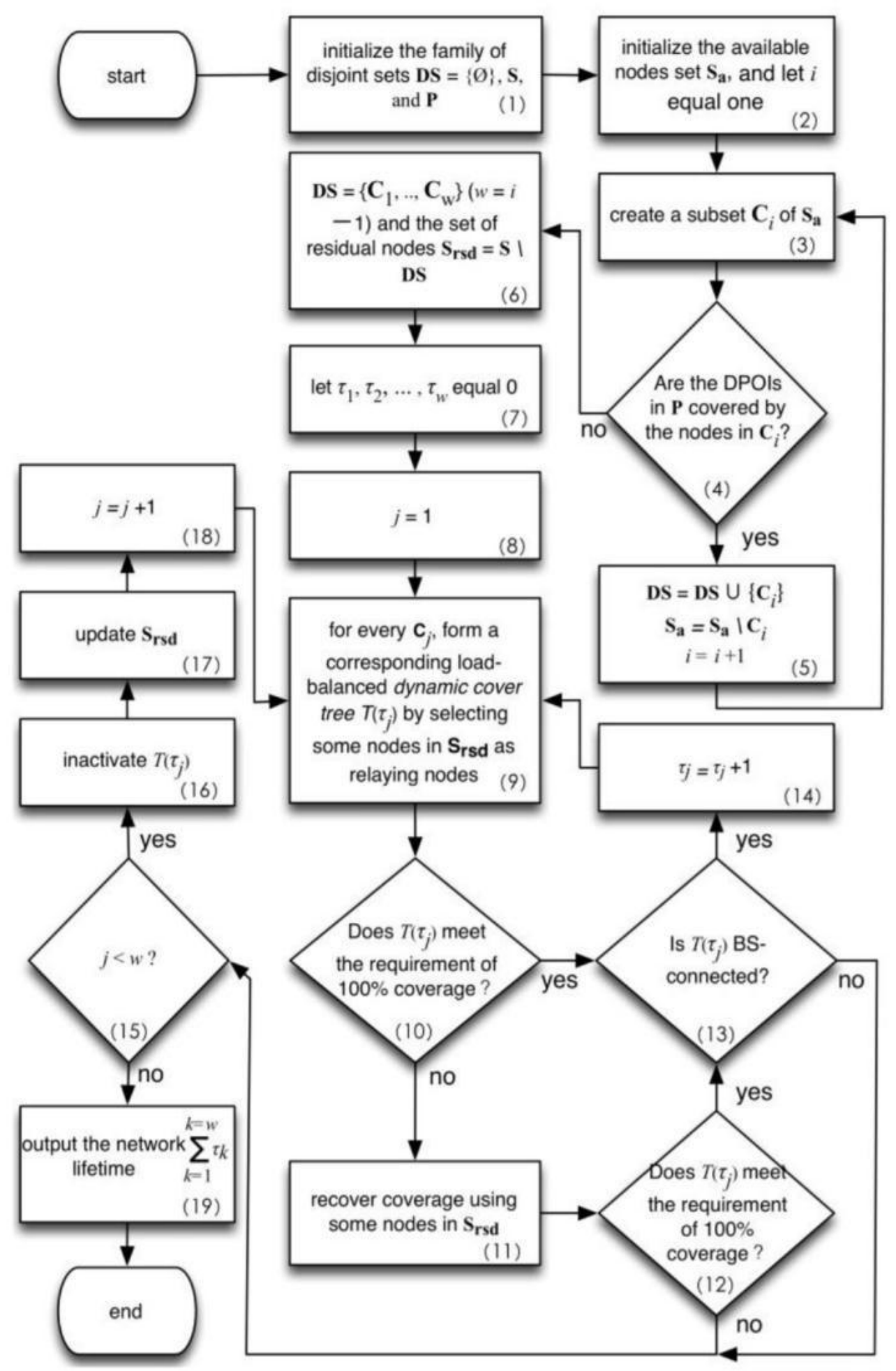

Fig -2: The Flowchart of the proposed Algorithm

\subsubsection{Dynamic Recovery for the Coverage Lost}

Numerous components which may impact the operation of WSN, including man made obstruction, climate change, and so forth. so it is fundamental to utilize an instrument to recoup the Typical working condition in the wake of starting the WSN. At the time when the full coverage fail, the hubs held in the arrangement of leftover hubs Srsd which can be used to recover the failed coverage of DPOIs (step11) with the help of "COP" algorithm. The nodes which are excluded from the disjoint set(step6) and currently available nodes were present in node-set Srsd. The hubs without adequate vitality to keep up a typical operations are wiped out from Srsd. Additionally, these hubs in Srsd are additionally seen as the applicant hubs to be chosen as the handing-off hubs. By thusly, the system lifetime of keeping up full scope can be effectively expanded. 


\subsubsection{Allowance of Link between Hubs in the Same}

\section{Tier}

On the off chance that the hub dependably transmits the detected information to a particular hub, the parent hub which may have heavier weight and quickly drain its energy, which facilitate to make the transmission way invalid. The invalid transmission way bring about the full coverage checking to come up short. Keeping in mind the end goal to stay away from such a circumstance, the sensor hubs need to build up associations with accessible neighbor hubs in the same level on the off chance that it has stand out competitor parent hub. Note that, the hubs are said to be in the same tier on the off chance that they have the same bounce tally number to the BS.

\subsubsection{Load Balance between the Nodes Present in} Same Tier

The sensor node which senses the data is always transmitted to the base station in every tree like topology. The data is transmitting which follow the direction from higher tier to lower tier. Whenever the node relaying the data it has to select a effective parent node. The given MCLCT make use of PLB identify the node probability for attempt made to transfer the data to each parent node. At the point when the hubs attempt to pick the best applicant parent hubs through the PLB procedure, they likewise build the by and large load-balanced dynamic cover tree. Along these lines, the system problem area what's more, blockage can be maintained a strategic distance from, and a more drawn out network lifetime.

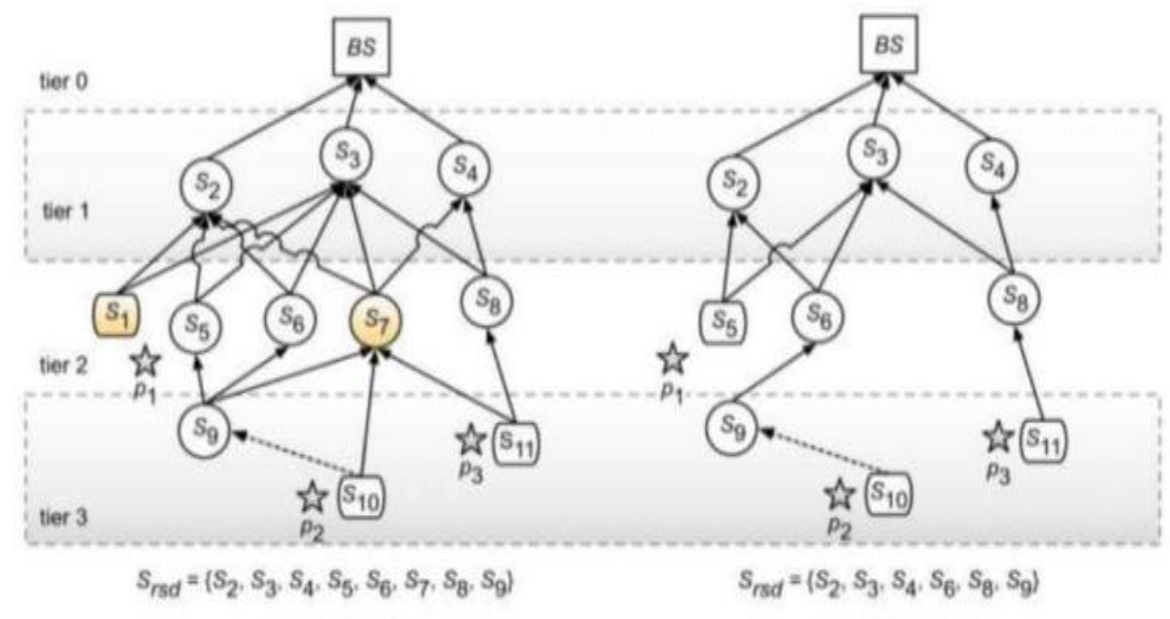

(a) $\pi(n$ at $r$

(b) $\pi(t)$ at $t+1$

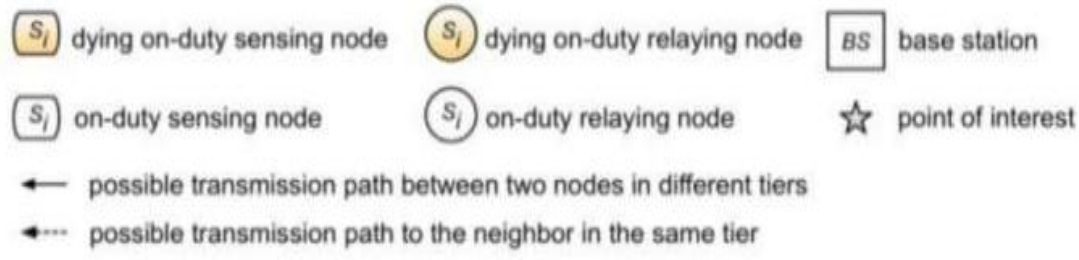

Fig-3 Demonstration of coverage recovery

\subsubsection{Stop Preforming Multiple Task at Same Time}

\section{Such as Relaying and Sensing}

The energy of every node in wireless sensor network is limited so if the node in network preform both sensing and relaying than the energy of the node drain rapidly and the network lifetime will be minimum. So every node supposed consider as a sensing or relaying node at a time but not both.

Figure 3 exhibits a basic illustration in regards to recovering the lost coverage. As shown in Figure 3a, at $\tau$, the Dynamiccover-tree $(\tau)$ comprises of 3 sensor device to sense the 3 DPOI, p1, p2, p3, and 8 relaying node which use to exchange the sensed data In subtle element, s1, s10, and s11 structure the disjoint set; ss2, s3, s4, s5, s6, s7, s8, and s9 structure the set Srsd. In the first place, the association in the middle of s9 and s10 is set up subsequent to s10 has a sole applicant parent hub (s7) in the lower (level 2). We assume that the detecting hub and transferring hub (i.e., s1 and s7) will drain their own particular energy.

\section{THE COVERAGE-OPTIMIZING-RECURSIVE}

\section{ALGORITHM}

The Coverage-optimizing-recursive algorithm is the first part of proposed MCLCT. The task of COR is to search the disjoint sets of node in the network. A node which located in every disjoin set $\mathrm{Ci}$ can be monitor all the DPOIs. By activating the disjoint-set alternatively in DS, can maximize the life time of the network and provide the full sensing coverage. The lost coverage of the wireless sensor network will be recovered by the COR algorithm to provide full coverage. The detial operation of COR heuristic is as follow 


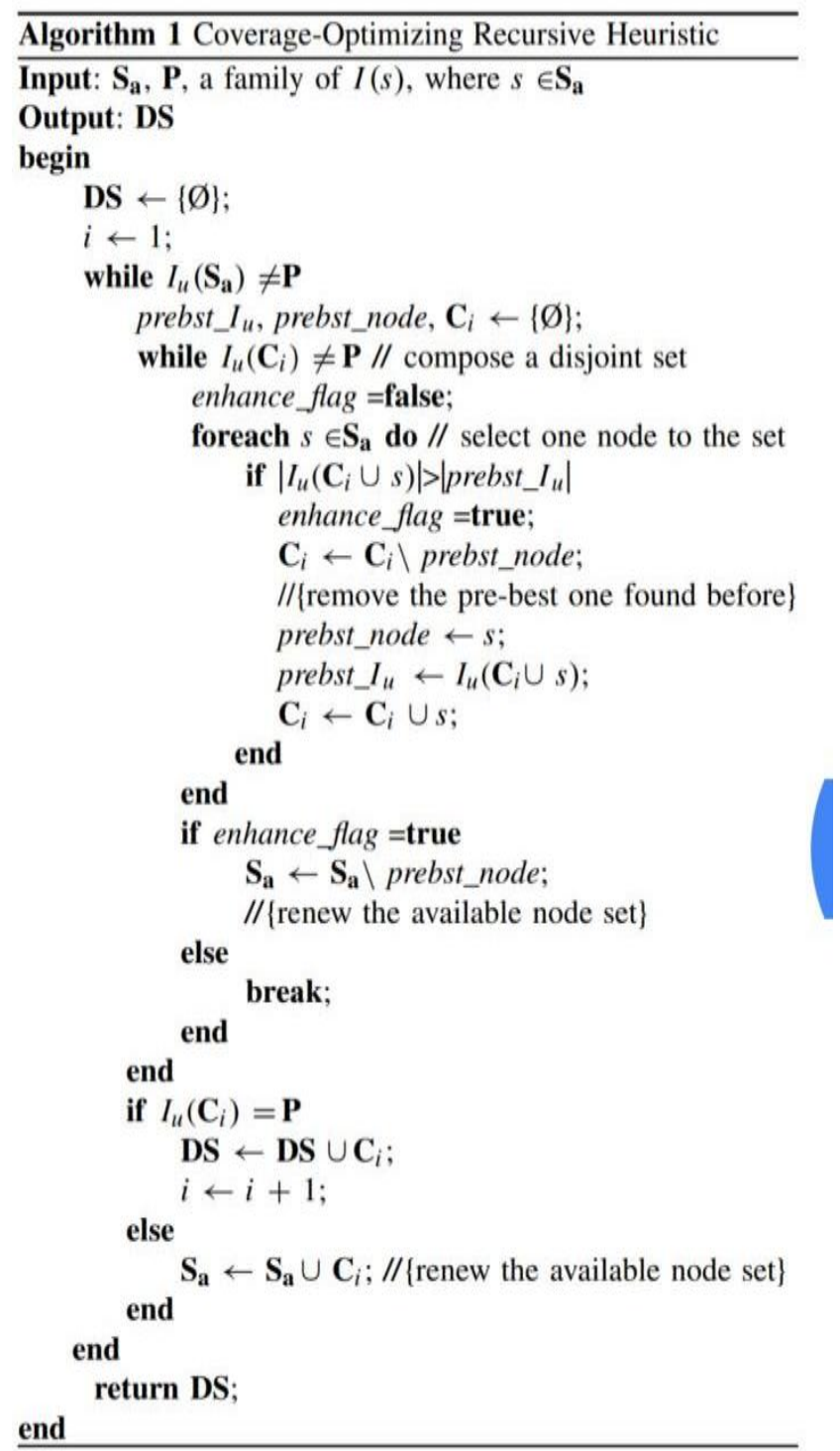

\section{THE PROBABLE LOAD BALANCING}

The task of the probable load balance (PLB) in the proposed algorithm is to maintain the load between the nodes by probable transmission of information from source to destination. Consider a random node si located in $\mathrm{k}^{\text {th }}$ tier. Suppose $\mathrm{v}$ is considered as a candidate parent node, which is represent by $\mathrm{Ptr}(\mathrm{Si})=\{\mathrm{sr} 1, \mathrm{sr} 2, . ., \mathrm{srv}\}$.

The probability of relaying the information from Si to parent node at $\tau^{\prime} \mid$ is as represented $\left\{{ }^{\sim} \mathrm{P}(\mathrm{si}, \mathrm{sr} 1, \tau),{ }^{\mathrm{P}}(\mathrm{si}, \mathrm{sr} 2, \tau)\right.$, . .. , ${ }^{\sim} \mathrm{P}(\mathrm{si}$, srv, $\left.\tau)\right\}$, where $0 \leq \sim \mathrm{P}(\mathrm{ci}, \operatorname{srq}, \tau) \leq(1 \leq \mathrm{q} \leq$ $\mathrm{v})$, and ${ }^{\sim} \mathrm{P}(\mathrm{si}, \operatorname{sr} 1, \tau)+{ }^{\sim} \mathrm{P}(\mathrm{si}, \operatorname{sr} 2, \tau), \ldots,+{ }^{\sim} \mathrm{P}(\operatorname{si}, \operatorname{srv}, \tau)$ $=1$.



\section{RESULT AND GRAPHS}

The tree topology with 16 nodes are used in figure 4. Where each node are in sleeping mode. When any information is sensed by the sensor node that sensor node generate a wakeup message to its selected parent node and the data is transmitted through a multi routing to balance the load between the nodes. Each node has its own cover set which consist of the directly connected nodes and the node which are not directly connected are known as disjoint nodes.

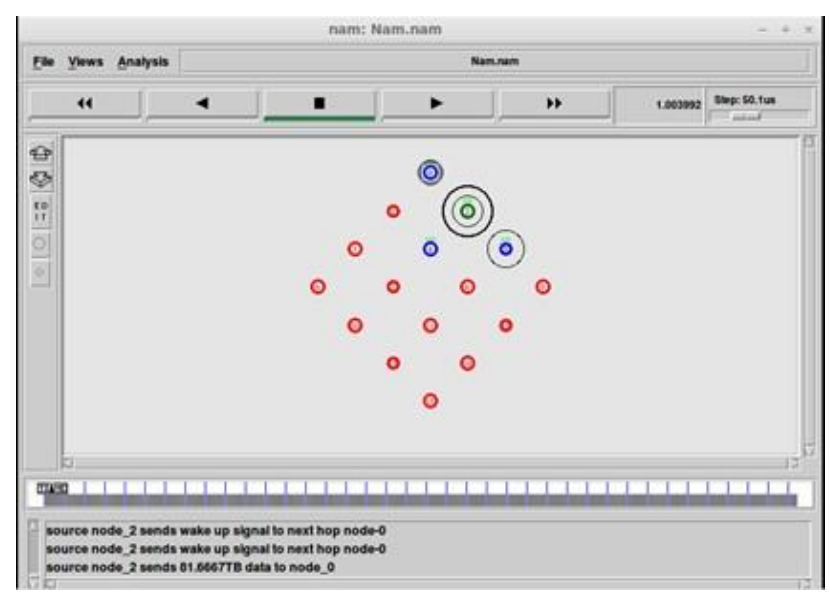

Fig-4 Simulation of MCLCT 
When the sensed data is transmitted to the base station. We need to check what amount of energy is consumed during the transmission of data. So the below graph show the total energy consumption during data transmission

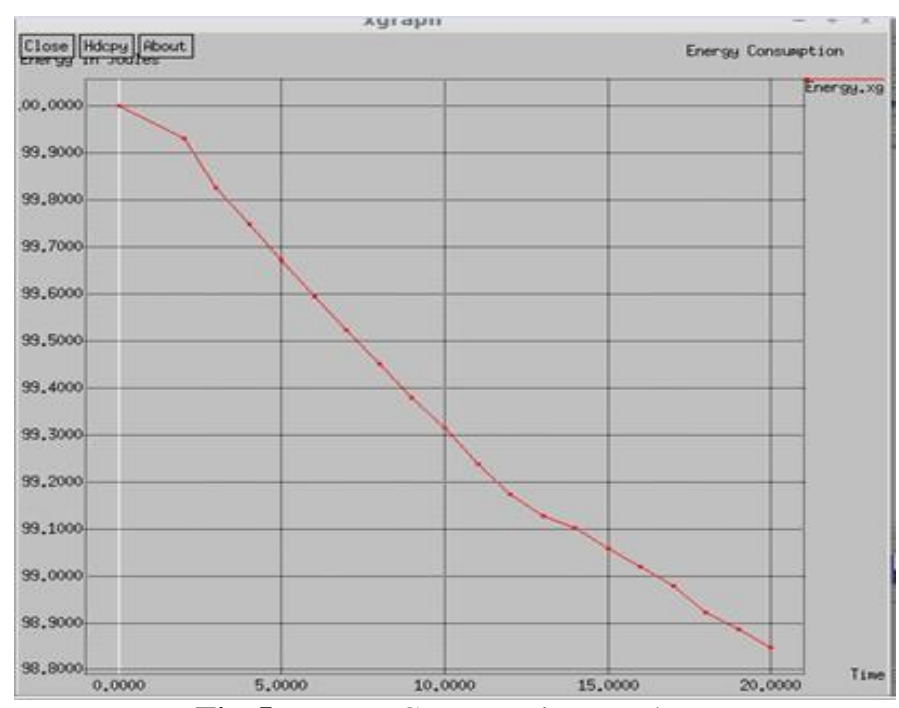

Fig-5 Energy Consumption graph.

\section{CONCLUSION}

The MCT issues are handled in this article by MCLCT algorithm. The aim of the MCT issues is to increse the life of the network. The proposed algorithm has two main part, the coverage optimization recursive and probable load balance. The "COR heuristic", can quickly locate a most extreme number of cover as indicated by the worldwide data of WSNs. Every cover set has some amount of sensing nodes. Thereafter the PLB technique progressively decide the best parent nodes to transfer sensed information. Those the energy productive operation can be accomplished by the MCLCT. Finally we shown the network topology of 16 nodes with the energy consumption graph.

\section{REFERENCES}

[1]. X. G. Cao,X. Han, E. L. Loyd, and C.-C. Shen, "Faulttolerant relay node placement in heterogeneous WSN," IEEE Trans. Mobile Comput., vol. 9, no. 5, pp. 643-656, May 2010.

[2]. R. Rajagopal, A. Krause, A. Gupta, and C. Guestrin,, "Simultaneous optimization of sensor placements and balanced schedules," IEEE Trans. Autom. Control, vol. 56, no. 10, pp. 2390-2405, Oct. 2011.

[3]. S. Misra,D. Yang, X. Fang, G. Xue, and J. Zhang, "Two- tiered constrained relay node placement in wireless sensor networks: Computational complexity and effective approximations," IEEE Trans. Mobile Comput., vol. 11, no. 8, pp. 1399-1411, Aug. 2012.

[4]. X. Chu,H. Liu, Y.-W. Leung, and R. Du, "Minimumcost sensor placement for required lifetime in wireless sensor-target surveillance network", IEEE Trans. Parallel Distrib. System., volume. 24, number 9, pp. 1783-1796, Sep. 2012.
[5]. J.-A. Jiang et al., "A distributed RSS-based localization using adynamic circle expanding mechanism," IEEE Sensors J., vol. 13, no. 10,pp. 3754-3766, Oct. 2013.

[6]. S. J. Gortler and Y. Zhu, "Sensor network localization using sensor perturbation," ACM Trans. Sensor Netw., vol.7, no. 4, p. 36, Feb. 2011.

[7]. J.-A. Jiang et al., "Collaborative localization in wireless sensor networks via pattern recognition in radio irregularity using omnidirectional antennas," Sensors, vol. 10, no. 1, pp.400-427, Jan. 2010.

[8]. M. Gurusamy and Q. Zhao, "Lifetime maximization for connected target coverage in wireless sensor networks," IEEE/ACM Trans. Netw., vol. 16, no. 6, pp. 1378-1391, Dec. 2008.

[9]. Cardei and I. CardeiM, "Energy-efficient connectedcoverage in wireless sensor networks," Int. J. Sensor Netw., vol. 3, no. 3, pp. 201-210, Jun. 2008.

[10]. M. Dehghan, P. Ostovari, and J. Wu, "Connected point coverage in wireless sensor networks using robust spanning trees," in Proc. 31st Int. Conf. Distrib. Comput. Syst. Workshops (ICDSC), Jun. 2011, pp. 287-293.

[11]. A. A. Abouzeid and N. Jaggi, "Energy-efficient connected coverage in wireless sensor networks," in Proc. 4th Asian Int. Mobile Comput. Conf. (AMOC), 2006, pp. 77-86.

[12]. C. Douligeris and D. Zorbas, "Connected coverage in WSNs based on critical targets," Comput. Netw., vol. 55, no. 6, pp. 1412-1425, Apr. 2011.

\section{BIOGRAPHIES}



Mr. MohammedJameel. A. Zarzari Completed BE computer science in SIET College Bijapur, Under VTU Belgaum, Now pursuing $M$. tech Computer Networking in TJIT college Bangalore, under VTU Belgaum

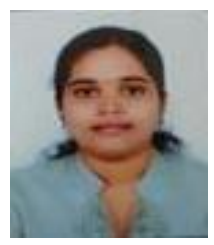

Ms. Bindhu. Madhavi: Designation: Associate Professor in TJIT Bangalore. Experience 09+years of Teaching and Areas of Interest: Wireless Sensor Networks, AdHoc Networks.

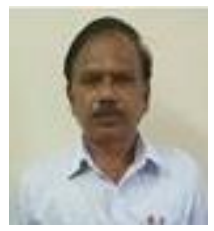

Dr. M. Nagendra, Designation Associate Professor in S.K University, Anantapur, Karnataka. 